\section{Manifest der SGP und der SGKJPP}

Duplik auf Ihr Schreiben vom 8. März 2001

Sehr geehrte Herren

Ich nehme Bezug auf Ihr Mail vom 8. März 2001 bzw. den darin enthaltenen Brief, der mich zu folgenden Bemerkungen, Feststellungen und Vorschlägen veranlasst:

1. Wie Sie wissen, ist die Kompetenz für die konkreten Tarifverhandlungen in die Hände der Tarifdelegation übergegangen. Meine Antworten, sofern nicht persönlicher Natur, beziehen sich ausschliesslich auf Ihre Anliegen an den Zentralvorstand.

2. Der Zentralvorstand hat im Tarifbereich folgende Aufgaben:

- Erarbeitung und Gerierung des UV-/MV-/IVTarifs;

- Erarbeitung einer Tarifstruktur für den KVGBereich;

- Unterstützung der ärztlichen Vertragsparteien bei Abschluss von Verträgen im KVG-Bereich.

Die Punkte 1 und 2 sind bis auf die Frage des Reengineerings (RE) erfüllt; die entsprechenden Vorlagen gehen zum Beschluss an die ordentliche Ärztekammersitzung im Mai 2001. Das RE hat begonnen; die Verzögerung ist auf die bis vor kurzem fehlende Bereitschaft der anderen Vertragsparteien zurückzuführen. Ihre Fachgesellschaften werden in den Prozess einbezogen werden (etwa April, Mai 2001).

Punkt 3 ist eine (an)dauernde Aufgabe, die sich durch die Bedürfnisse der Vertragsparteien bestimmt.
3. Ich verzichte auf eine Wiederholung unserer Positionen $\mathrm{zu}$ Ihren schon vorgängig gemachten Forderungen und Bedenken; Neues ist hier nicht hinzuzufügen. Der Ordnung halber halte ich fest, dass im Einflussbereich des ZV Ihre Forderungen erfüllt sind, wie Ihnen die Vorlagen an die Ärztekammer zeigen werden. Dies gilt insbesondere für Ihre Forderung nach einem Stundenumsatz von mindestens Fr. 200.-.

4. Es ist, und dies gerade auf Punkt 3 bezogen, ein ebenso bekanntes wie einfaches Vorgehen, missliebigen Fakten nicht in die Augen sehen zu müssen, in dem man sie mit dem Killerargument der "Unhöflichkeit» aus seinem kognitiven Bereich eliminiert.

5. Sie haben um ein Gespräch mit dem ZV und der Tarifdelegation nachgesucht. Einzige Möglichkeit, dies vor der ordentlichen Ärztekammersitzung zu realisieren, bietet die nächste ZV-Sitzung vom 24. März 2001. Wir laden Sie auf dieses Datum, 15.30 Uhr bis max. 16.45 Uhr, zu einer Besprechung in Anwesenheit der Tarifdelegation nach Bern ein; die formelle Einladung wird Ihnen separat zugehen.

6. Wir werden, Ihr Einverständnis voraussetzend, Ihre und unsere Duplik in der Schweizerischen Ärztezeitung publizieren. Mit freundlichen Grüssen
FMH

Dr. H. H. Brunner Präsident 